\title{
The Optical Design of a Far Infrared Imaging FTS for SPICA
}

\author{
Carmen Pastor ${ }^{1}$, Pablo Zuluaga ${ }^{1}$, Willem Jellema ${ }^{2}$, Luis Miguel González Fernández ${ }^{1}$, Tomas \\ Belenguer ${ }^{1}$, Josefina Torres Redondo ${ }^{3}$, Peter Paul Kooijman ${ }^{2}$, Francisco Najarro ${ }^{3}$, Martin Eggens ${ }^{2}$, \\ Peter Roelfsema ${ }^{2}$, Takao Nakagawa ${ }^{4}$ \\ ${ }^{1}$ Instituto Nacional de Técnica Aeroespacial (INTA), Carretera de Ajalvir Km 4.5, 28850 Torrejón \\ de Ardoz, Madrid, Spain; ${ }^{2}$ SRON Netherlands Institute for Space Research, Department of Low \\ Energy Astrophysics, P.O. Box 800, 9700 AV Groningen, The Netherlands; ${ }^{3}$ Centro de \\ Astrobiología (INTA-CSIC), Carretera de Ajalvir Km 4.5, 28850 Torrejón de Ardoz, Madrid, \\ Spain; ${ }^{4}$ Japan Aerospace Exploration Agency (JAXA), Institute of Space and Astronautical Science \\ (ISAS), Department of Infrared Astrophysics, 3-1-1 Yoshinodai, Chuo-ku, Sagamihara, Kanagawa \\ 252-5210 Japan
}

\begin{abstract}
This paper describes the optical design of the far infrared imaging spectrometer for the JAXA's SPICA mission. The SAFARI instrument, is a cryogenic imaging Fourier transform spectrometer (iFTS), designed to perform backgroundlimited spectroscopic and photometric imaging in the band 34-210 $\mu \mathrm{m}$. The all-reflective optical system is highly modular and consists of three main modules; input optics module, interferometer module (FTS) and camera bay optics. A special study has been dedicated to the spectroscopic performance of the instrument, in which the spectral response and interference of the instrument have been modeled, as the FTS mechanism scans over the total desired OPD range.
\end{abstract}

Keywords: far infrared, optical design, iFTS, interferometry, diffraction analysis

\section{INTRODUCTION}

SpicA FAR infrared Instrument, SAFARI, is an imaging spectrometer which is being designed to map large areas of the sky in the far infrared. The SPICA mission, having a large cold telescope cooled to $6 \mathrm{~K}$ above absolute zero, will provide an optimum environment where instruments are limited only by the cosmic background itself. [2]

The optical design of an instrument working for the far infrared and involving interferometry at the same time, as in our case for SAFARI, is quite challenging and must pay great and dedicated attention to at least three main design working areas on top of the base geometrical optical design; areas such as the propagation \& diffraction analysis, the interference modeling of the FTS scanning, and the baffling strategy. The first area of consideration is dedicated to the beam diffraction effects, which must be unavoidably taken into account, as the working wavelength is considered to be large compared with the size of the optical components. On the other hand, the interference modeling of the FTS while scanning over the total desired OPD range, will give us important information about the spectral response of the instrument as built, even taken into account the manufacturing and alignment tolerances. The third area of study would be the stray light control, as all the optical components and mechanical parts that are warmer than a few kelvin are highly self-luminous at these wavelengths, and they are therefore potential spurious signal contributors to the sometimes faint astronomical sources of interest. We will not cover the SAFARI baffling strategy in this paper, but we are keeping in mind the future need of providing sufficient room around the beam and mounts for stray-light baffles and oversized apertures. The general scope of this paper is to present the conceptual or reference optical design and its current status. CODEV and MatLab have been the main software packages we have used for the design and analysis of this optical system.

Space Telescopes and Instrumentation 2014: Optical, Infrared, and Millimeter Wave, edited by Jacobus M. Oschmann, Jr., Mark Clampin, Giovanni G. Fazio, Howard A. MacEwen, Proc. of SPIE Vol. 9143, 91434B · @ 2014 SPIE

CCC code: $0277-786 \mathrm{X} / 14 / \$ 18 \cdot$ doi: $10.1117 / 12.2056791$ 


\section{SAFARI KEY REQUIREMENTS}

The optical design of SAFARI will provide imaging spectroscopy for the sky signal coming through the SPICA telescope and into the SAFARI optics. The main SAFARI optical requirements derived from the Science Requirements are [2]:

a) The instrument shall provide direct imaging, with diffraction limited capability in three spectral bands over 34$210 \mu \mathrm{m}$ and over a 2'x2' FoV on sky.

b) The three wavelength bands covered by SAFARI are:

SW - Short Wave, 34-60 $\mu \mathrm{m}$

MW - Medium Wave, 60-110 $\mu \mathrm{m}$

LW - Long Wave, 110-210 $\mu \mathrm{m}$

c) The instrument shall implement a FTS based spectroscopy mode with spectral resolving power up to $\mathrm{R}=2000$ at $100 \mu \mathrm{m}$ over the required FoV.

d) The instrument shall have an optical interface with the SPICA Telescope defined as an incoming beam of F/5.4 and $16.2 \mathrm{~m}$ focal length, $3 \mathrm{~m}$ entrance pupil aperture, being the aperture stop at the secondary telescope mirror (M2). The signal will be picked by a pick-off mirror at a nominal field position of 7' off-axis.

\section{FUNCTIONAL BLOCKS OF SAFARI}

The instrument functional blocks layout is shown in Figure 1. The figure does not represent the exact layout of the instrument, as it has been spread for better visualization of the three modules.

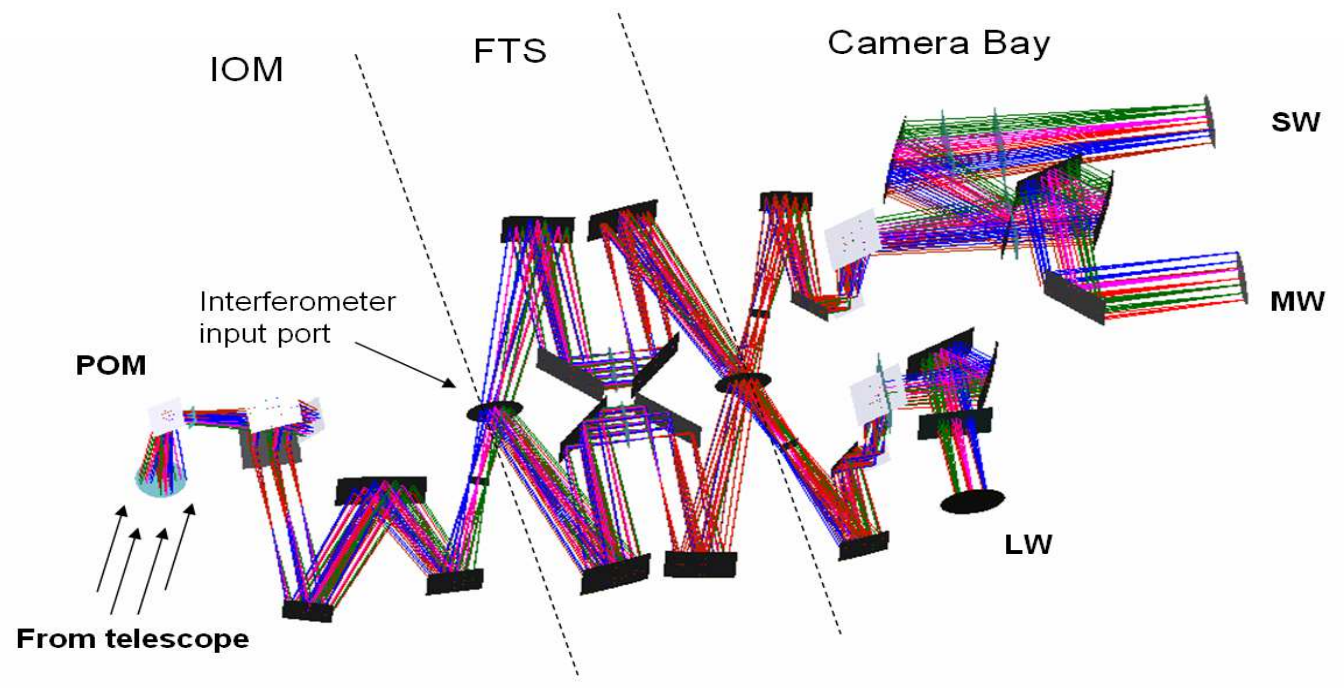

Figure 1. SAFARI Instrument Functional Blocks.

As shown in the figure, radiation coming from the telescope enters the instrument via the pick-off mirror (POM) and into the Input Optics Module (IOM). After focusing at an intermediate image plane, the radiation enters the interferometer optics, previously split into two arms by the beamsplitter located at the first input port of the interferometer. The interferometer generates two output ports, each of them going through a dedicated filter wheel, one for the SW and MW bands and another one for the LW band. After undergoing the desired interference the two beams reach the Camera Bay optics, where the radiation is focused into the LW, MW and SW Focal plane arrays. The Calibration source radiation (not shown in Figure 1) enters the instrument via the second input port of the interferometer, utilizing a dedicated optical system that produces a flat field at the final image plane. 


\section{OPTICAL DESIGN DESCRIPTION}

The optical design of SAFARI is all reflective, (with the exception of the beam/splitters and filters), and consists of three main optical modules (plus the calibration source optics):

Input Optics Module, interferometer Optics (or FTS Optics) and Camera Bay Optics.

The three modules are finite conjugate optical systems and follow the same optical concept, relay optics systems consisting of two powered off axis mirrors and a pupil image in between the mirrors. Each optical module in the SAFARI optical design consists then of a collimating mirror, which produces a real pupil image in between the powered mirrors and a focusing mirror at the rear of the optical system to achieve the desired F/\#. The three modules can be aligned and verified separately, as they are all relay optics and attend to different optical functions within the whole optical design. A block diagram is shown in Figure 2. The three modules shall be designed to operate at a temperature of 4.5K. Inside the Camera Bay Optics there will be a structurally separated compartment, named Cold Box, which shall be design to operate at $1.7 \mathrm{~K}$. In the diagram only the powered mirrors are shown. All powered mirrors are conical.

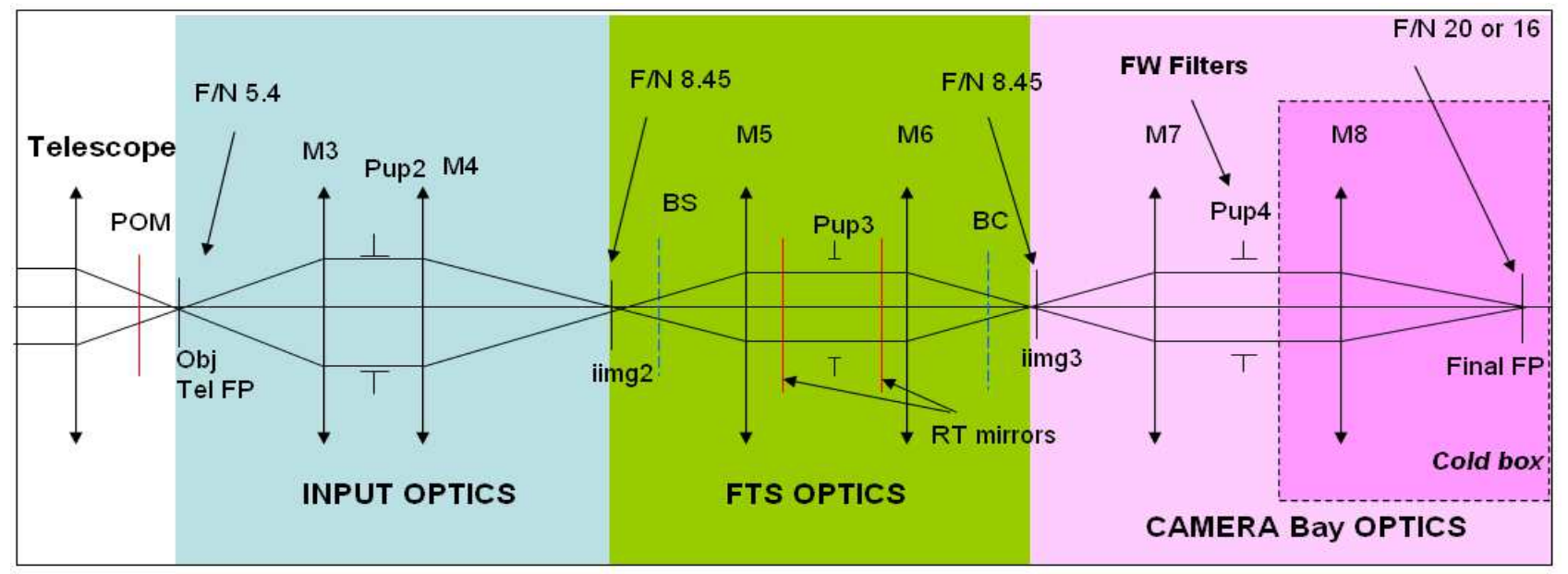

Figure 2. Blocks diagram of the Safari optical design.

\subsection{Paraxial Optical Model}

The specification of the SAFARI optical design requires appropriate control of both the intermediate scene images and the intermediate pupil images in the system. The correct distribution of the magnification of the different subsystems or modules within the instrument guarantees a deeper control of the modules individual performance and their function in the whole instrument. For the sake of simplicity and in order to have a close control of the main construction parameters, we have built a "paraxial optical model" of the instrument which provides immediate information of the modules behavior and their contribution to the final image specification. The "paraxial optical model" is an all-centered optical system simulating all the mirrors, pupils and intermediate images in SAFARI. The model consists of two zoomed positions, one that accounts for the scene image formation and the other one that accounts for the pupil image formation. An initial optimization will give us quick information of the location and size of the different field and pupil images as information of the different mirrors focal lengths and even provides results out of a quick beam propagation analysis that accounts for the different apertures of the optical elements in the optical train, including the telescope mirrors apertures and the spider. In the following sections a description of the design details of each of the functional modules is being provided.

\subsection{Input Optics Module}

The functions of the Input Optics Module (IOM) are to provide:

a) Optical interface with the SPICA telescope at the IOM object space, receiving a beam with an aperture F/5.4, and a 
telescope focal plane with certain amount of field curvature. The telescope beam is redirected onto the SAFARI instrument via the pick off mirror at an offset of the FoV of 7 arc minutes.

b) Optical interface with the Interferometer (FTS Optics) at the IOM image plane, feeding the FTS optics with a beam $\mathrm{F} / 8.45$.

c) An accessible pupil image in transmission for appropriate beam steering.

The IOM contains 7 mirrors including the POM. It contains 2 powered mirrors, the rest of the optics are folding mirrors required to keep the overall volume of the instrument as compact as possible. The telescope beam is picked up before the focal plane image which provides internal access to the focal plane. The entrance pupil of the SAFARI instrument is the SPICA Telescope secondary mirror (M2). The IOM provides an internal and accessible pupil image of M2. The IOM is a relay optics system with magnification of about $1.57 \mathrm{X}$ re-imaging the telescope focal plane with F/5.4 to an output image in front of the interferometer optics with an aperture of F/8.45. In Figure 3 the optics of the IOM is shown.

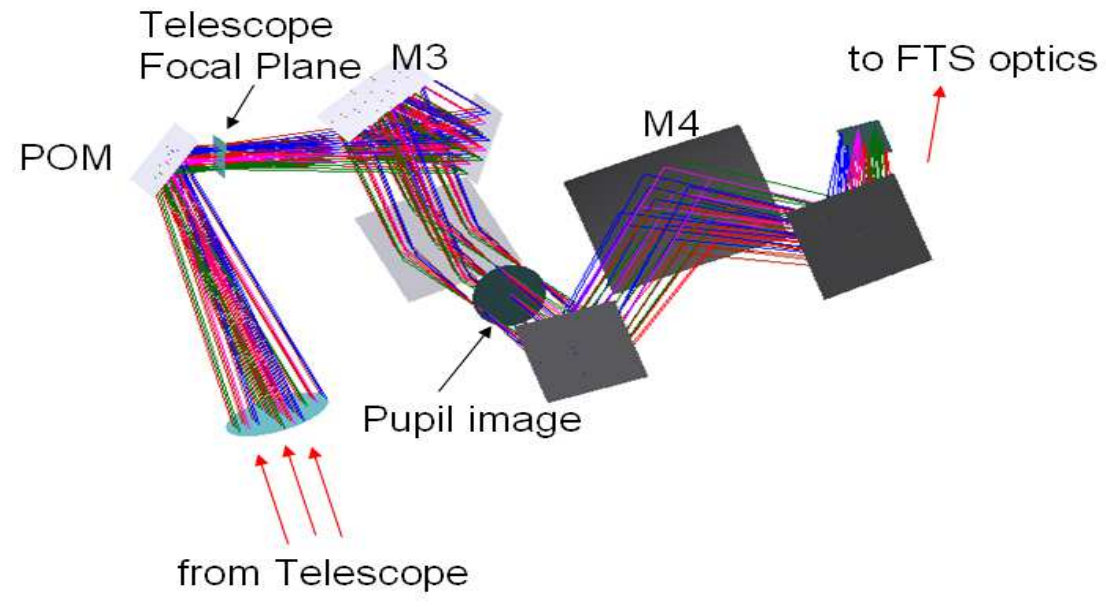

Figure 3 Input Optics Module Layout

\subsection{Interferometer Optics}

The functions of the Interferometer optics are to provide:

a) Optical interface with the IOM receiving a beam F/8.45, and a flat intermediate image.

b) Optical interface with the Camera Bay Optics at the FTS image plane, feeding the Camera Bay Optics with a beam $\mathrm{F} / 8.45$, and producing a flat image plane.

c) A real pupil image in transmission in between the roof-top mirrors.

d) Spectral resolving power at the medium band $(100 \mu \mathrm{m})$ of $\lambda / \Delta \lambda=2000$

The interferometer optics accepts the F/8.45 beam from the IOM and is also a relay optics system with magnification 1X. The configuration consists of two identical arms corresponding to both arms of the Mach Zehnder interferometer. The optical system consist of a collimating mirror M5 forming a pupil image at the centre of the rooftop mirror configuration of the FTS mechanism. The interferometer optics is required to be symmetric in the pupil of the Fourier Transform Spectrometer Mechanism (FTSM) for zero Optical Path Difference (OPD). The FTS optics are made up of 4 conical mirrors (2xM5 and 2xM6) and contain the beam-splitter and beam-combiner optical elements. There are also 4 flat mirrors (roof-top mirrors) that configure the complete Mach Zehnder configuration. The configuration of the interferometer provides a folding of a factor of 4 in the OPD with respect to the actual movement of the mirrors, this means that the total range of movement of the mirrors will provide a total OPD of $140 \mathrm{~mm}$.

In Figure 4 the layout of the interferometer optics is shown. 


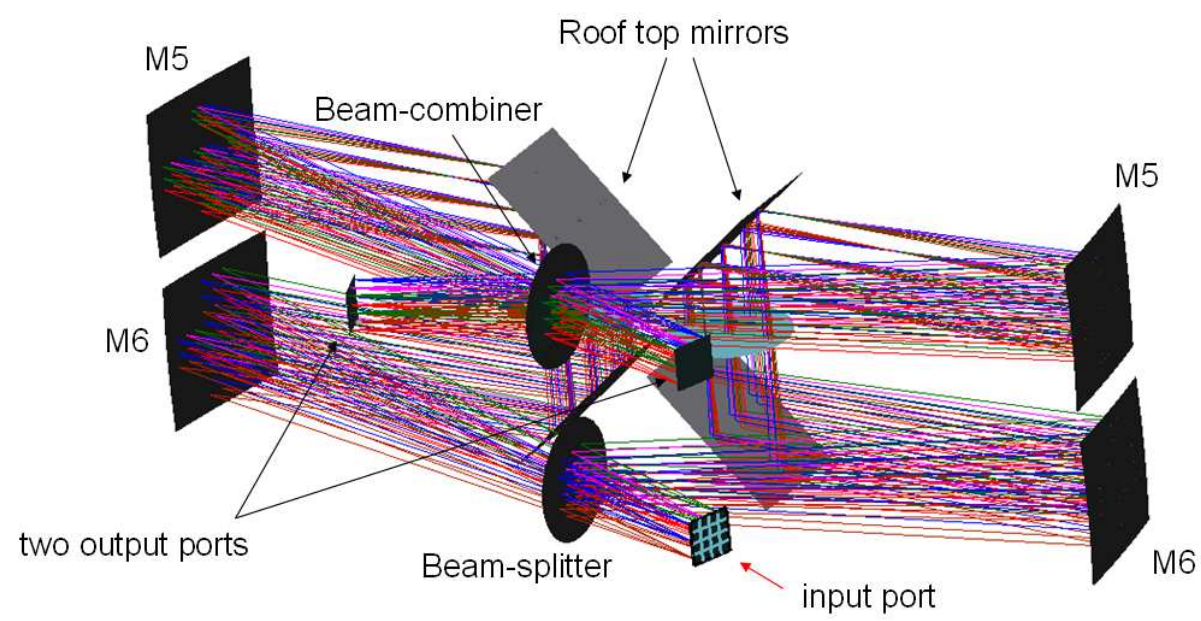

Figure 4.Layout of the Safari interferometer optics.

\subsection{Camera Bay Optics}

The functions of the Camera Bay Optics are to provide:

a) Optical interface with the Interferometer Optics at both FTS output ports, one output port for the LW band and another output port for the SW \& MW band, receiving a beam F/8.45 at the intermediate images, produced both in transmission and in reflection right after the Beam Combiner.

b) A final image for each of the three bands, LW, MW and SW, at the focal plane arrays (FPA) location, with final aperture of F/20 for the SW and MW bands, and F/16 for the LW band.

c) An accessible pupil image in transmission for each output port, where the Filters and cold stop will be located.

The final part of the optical train is formed by the camera bay optics which starts at a field image in the output of the interferometer. The interferometer provides two output ports. The LW band is located in one output port, whereas the SW and MW bands are located in the other port. Each output port has its own dedicated filter wheel, meaning that one common filter wheel is available for the combined SW/MW band, and another independent filter wheel for the LW band. The filter wheels are positioned in the vicinity of a pupil image which sits at the entrance of the cold detector box. This pupil image is formed by the powered mirror M7 following the image plane of the interferometer. In Figure 5 the layout of the Camera bay optics is shown.

Inside the detector box there are mainly folding mirrors and for each band one powered mirror M8 providing the $\mathrm{f}$ number interface for the FPA's. There are 14 mirrors in total in the Camera bay optics, 5 powered and 9 folding mirrors. The needed packaging arrangement of the FPA's have lead to the need of that number of folding mirrors. The dichroic in the SW/MW band, reflects the short wavelength radiation, and is used at an angle of incidence below $22.5^{\circ}$. The final image pupil is virtual and nearly telecentric, being the degree of telecentricity different for each band. 


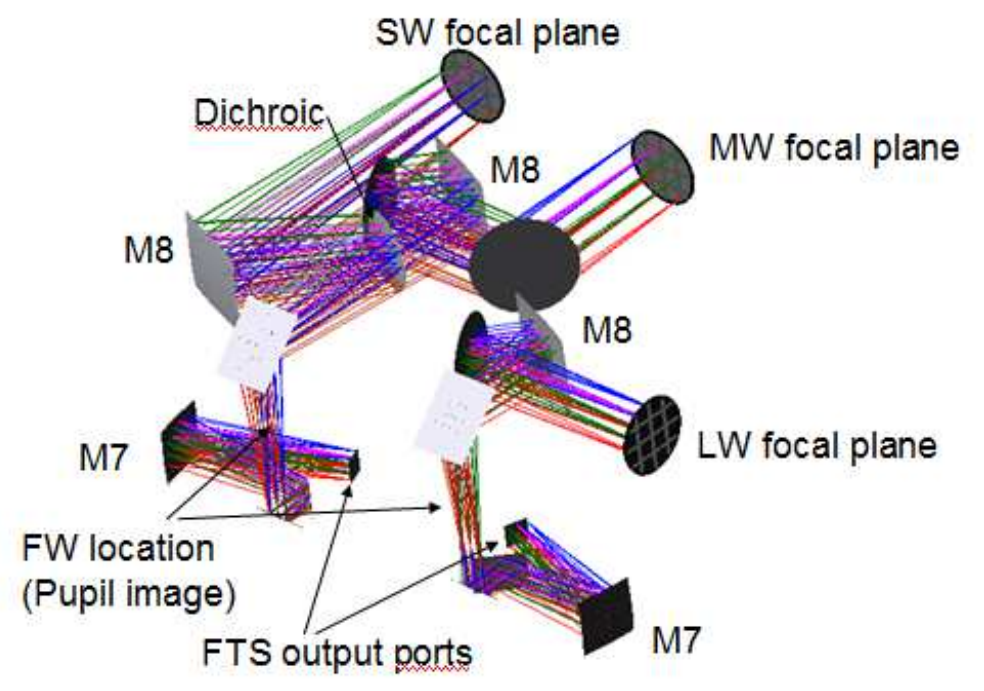

Figure 5. Camera Bay Optics.

\section{PROPAGATION AND DIFFRACTION ANALYSIS}

As mentioned in the introduction, as the working wavelength of SAFARI is large compared with the size of the optical components, it becomes important to model diffraction effects that occur all along the optical system, and not only consider the conventional "exit pupil diffraction model". A simulation that can account for the wave nature of light throughout the optical system or a beam propagation simulation is then mandatory for SAFARI. The main consideration would be that in our case the diffraction effects occur before the exit pupil, and can be not negligible for some of the surfaces or mechanical structures in our optical system. Methods that can account for the wave nature of light throughout all the surfaces in an optical system are referred to as beam propagation methods.

The modeling can be done with CODEV BSP tool (Beam Synthesis Propagation) to simulate the SAFARI spectroscopic and imaging performance. BSP is a Diffraction Propagation Tool that uses beamlet-based diffraction propagation and includes effects of aperture clipping, intermediate image structure, and lens or mirror aberrations.

As a preliminary and fast run we have used our paraxial optical model in which we have included the SPICA telescope, containing a possible configuration of the spider on M2, and the rest of the optical elements that complete the three SAFARI optical modules, input optics model, interferometer optics and camera bay optics.

This preliminary run will give us an idea of the critical elements that most affect the final signal, and the possible solutions for it. Resizing of the elements above their geometrically calculated clear apertures will be the most expected solution or mitigation of the diffraction effects assessment. At this point it becomes important to iterate with the straylight analysis for which the resizing of the elements can be quite critical for the spurious signals control and final baffling strategy. A compromise between these two disciplines will be necessary in a near future.

In Figure 6 the results of the preliminary propagation analysis for radiation of $160 \mu \mathrm{m}$ and some of the surfaces in the optical train is shown. The diffraction effects on the spider structure are quite noticeable on the shape of the PSFs for the intermediate images (see figure 6, b). The graphs show the intensity (in $\mathrm{dB}$ ) for some of the surfaces. As an explanatory example we should compare the geometrical clear aperture of the telescope focal plane, corresponding to a FOV of \pm 1 arcmin, which is $\pm 4.71 \mathrm{~mm}$, and the extent of the PSF at this surface which is, and just for an on axis beam, the one shown in Figure 6, b). We can observe a large spreading of the intensity, so the sizing of the surfaces, in particular the 
field stops, are highly critical in the final throughput of the optical system. Please note the representation of the intensity in the graphs is in decibel $(\mathrm{dB})$ scale.

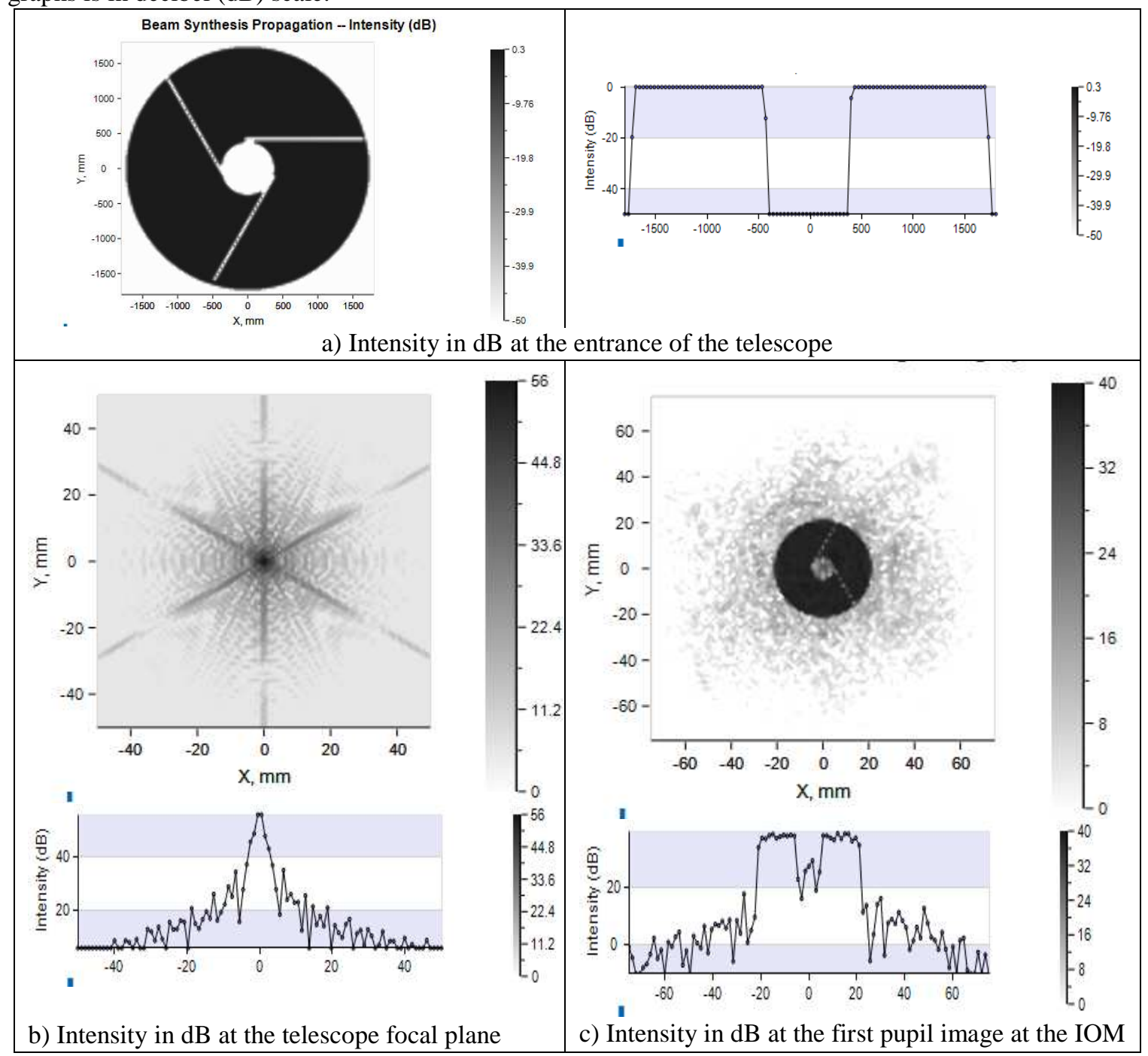

Figure 6. Preliminary Propagation Analysis for wavelength $160 \mu \mathrm{m}$. Field Intensity in $\mathrm{dB}$

\section{INTERFERENCE SIMULATION}

The spectroscopic performance of SAFARI can be affected by several factors related with the diffraction effects of the radiation for the instrument wavelengths; among those factors are the guiding accuracy of the FTS scan, and the pupil wandering of the system as the FTSM (FTS Mechanism) scans over the total desired OPD range.

The interference simulation of the FTS as operating over the total OPD scanning can also be modeled with the CODEV BSP tool. The outline of this simulation is as follows:

For each channel (FTS arm), the Complex Field at the image plane is computed. Then a subtraction of both channels Complex Field data is performed, simulating the interference between both arms (CODEV does not compute coherent propagation directly). Then a computation of the intensities for the interference pattern is performed and this is done for the whole FTS scan. We can reproduce then the real or as built optical system scanning and therefore its interferometrical or spectroscopic behavior. 
Each run of the simulation is a monochromatic run, so the running time is dependent on the number of wavelengths or bandwidth of the simulation

An example of output of this modeling is shown in Figure 7and Figure 8.

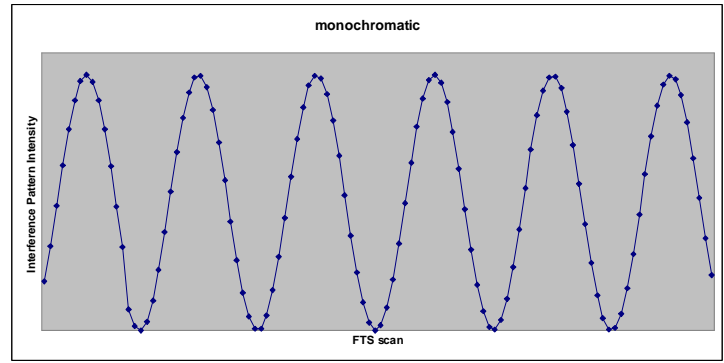

Figure 7. Interference modeling for the FTS scanning in SAFARI optical system (monochromatic).

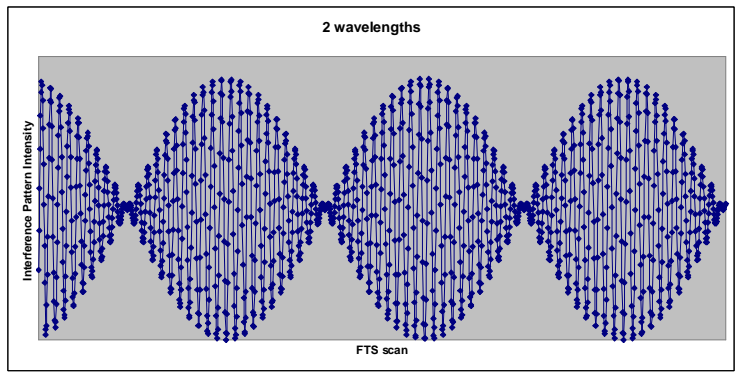

Figure 8. Interference modeling for the FTS scanning in SAFARI optical system and for 2 discrete wavelengths.

With this simulation we have also been able to reproduce the interferogram intensity pattern apodization that results for an off axis beam radiation as the OPD is scanned. In Figure 9 and Figure 10, the interferogram intensity patterns for an on-axis and off-axis beam respectively are shown. We can observe some decrease of the signal in the case of an offaxis beam, as the scan reaches its end. This is due to the pupil wandering which is produced in the FTS optical module as the OPD is swept. Other types of apodization or signal behavior could be studied with the help of this interference model. The retrieval of the modeled wavelengths, calculated as the FFT of the interferogram intensity patterns, are shown on the right of each figure. The model will also allow us to assess the effects of manufacturing and alignment tolerances on the spectroscopic performance of SAFARI
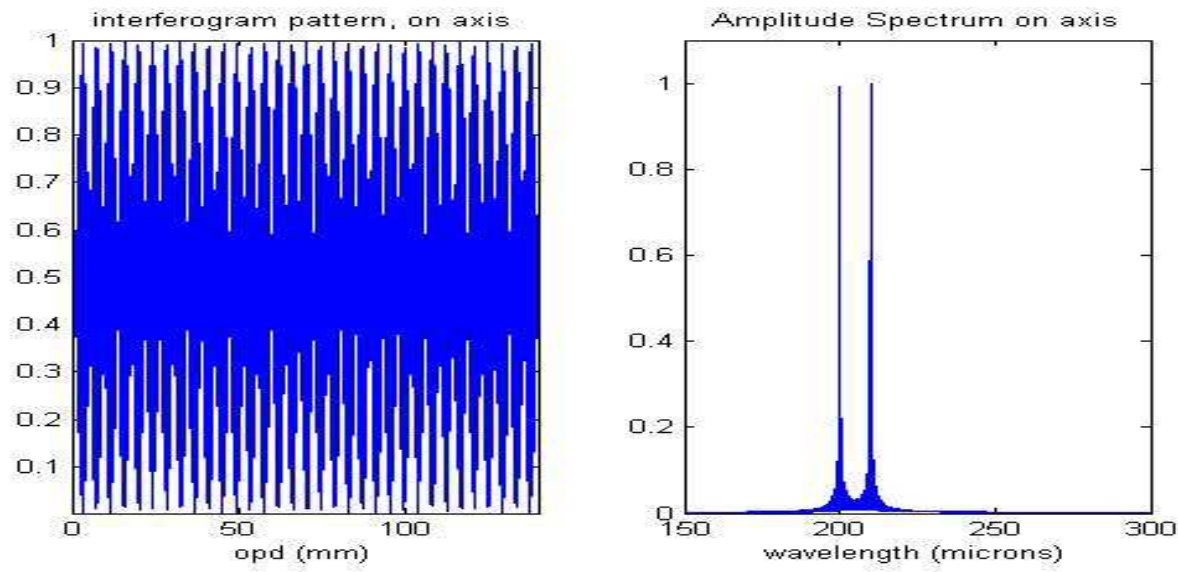

Figure 9. On axis Interference pattern @ $200 \mu \mathrm{m} \& @ 210 \mu \mathrm{m}$ (left), and its FFT (right) 

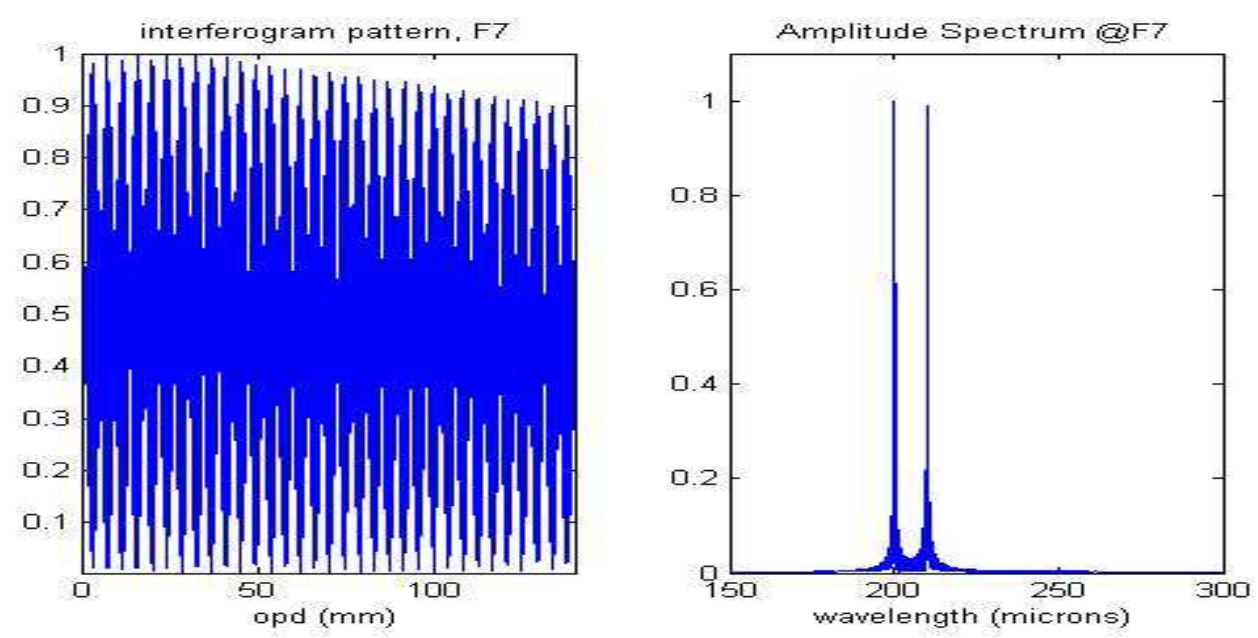

Note: F7 in Figure 10 represents an off axis extreme field point

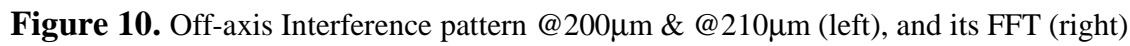

\section{CALIBRATION SOURCE OPTICS}

The Calibration Source optical system has been thought to produce a flat field on the FPA image plane. The optical system consists of a beam expander and a focusing mirror, plus some folding mirrors. The Calibration source will be an integrating sphere producing the desired radiance scenarios and for the SAFARI working wavelengths.

The integrating sphere is located at the focal plane of the beam expander 1st mirror, producing an image at infinity of the output port of the integrating sphere at every focal intermediate image plane, and consequently an image at infinity at the final image plane (flat field). The optical systems consists of a two mirror beam expander followed by two flat mirrors and a focusing mirror to guide the radiation coming from the output port of the integrating sphere into the second input port of the interferometer and through the rest of the optical train up to the final image plane. The study of the performance of this optical system is pending of further study with ASAP (Advanced Systems Analysis Program). In Figure 11, a layout of the optical design for the optics of the Calibration Source is shown.

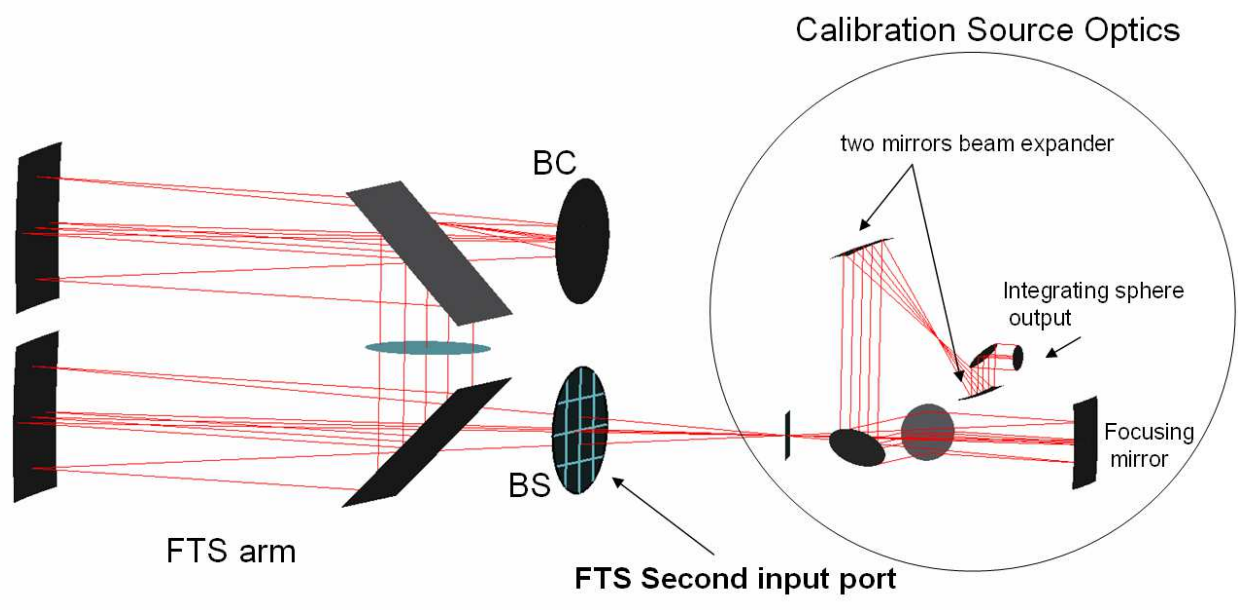

Figure 11. Calibration Source Optics. 


\section{CONCLUSION}

Currently we have achieved an Optical Reference Design which is to be optimized to comply with the final required specification.

The subunits optomechanical interfaces have been updated and the optical design fits the current available volume.

Each module (IOM, FTS \& Camera Bay) consists of 2 powered mirrors and an accessible pupil image in transmission in a collimated space, and no intermediate extra images are included in any the modules.

The SPICA Telescope interface has been updated and included in the optical design, and in the performance analysis.

The calibration source optical concept has been defined and is to be optimized to comply with the final required specification.

A paraxial optical model of the instrument has been designed to provide a quick feedback and preliminary assessment of the diffraction properties of the beam as it passes through the system, which alerts about providing some oversizing of the mirrors.

A simulation of the interference at the FTS has been developed for the system as built, in order to assess the spectroscopic performance of the instrument.

\section{ACKNOWLEDGMENT}

This material is based upon work supported and funded by Spanish MINECO under Grant Nr. FIS2012-39162-C06-03. The authors wish to thank TNO Optics in the Netherlands and SFTC-RAL Space in the UK for their initial contributions to a starting point optical design of SAFARI, in which the current reference design is based.

\section{REFERENCES}

[1] W. Jellema, B. Kruizinga, H. Visser, T.van den Dool, C. Pastor, J. Torres, M. Eggens, M. Ferlet, B. Swinyard, K. Dohlen, D.Griffin, L.M. Gonzalez, T. Belenguer, H. Matsuhara, M.Kawada, Y. Doi. "The optical design concept of SPICA-Safari” Proc. SPIE Vol. 8442 Space Tel. \& Instrum, Sep 2012.

[2] P. Roelfsema, M. Giard, F. Najarro, K. Wafelbakker, W. Jellema, B. Jackson, B. Swinyard, M. Audard, Y. Doi, M. Griffin, F. Helmich, F. Kerschbaum, M. Meyer, D. Naylor, H. Nielsen, G. Olofsson, A.Poglitsch, L. Spinoglio, B. Vandenbussche, K. Isaak, J. R. Goicoechea. "The SAFARI Imaging Spectrometer for the SPICA space observatory” Proc. of SPIE Space Tel. \& Instrum, Sep 2012

[3] B. Swinyard, P. Ade, M. Griffin, K. Dohlen, J.Baluteau, D.Pouliquen, D.Ferand, P. Dargent, G. Michel, J. Martignac, L. Rodriguez, D. Jennings, M. Caldwell, A. Richards, P. Hamilton, D. Naylor "The FIRST-SPIRE Spectrometer A Novel Imaging FTS for the Sub-Millimetre" Proc. SPIE 4013.Germany, 2000. 